\title{
Interactive and Intelligent Approach for Brain Extraction from High- Resolution Volumetric MR Neuroimages
}

\author{
Guoyu Qian ${ }^{1,2}$, Suhuai Luo ${ }^{1}$, Jesse Jin ${ }^{1}$, Mira Park ${ }^{1}$, Wieslaw L. Nowinski ${ }^{2}$ \\ ${ }^{1}$ School of Design, Communication and IT, The University of Newcastle, Australia \\ ${ }^{2}$ Biomedical Imaging Lab, Agency for Science, Technology and Research, Singapore
}

\begin{abstract}
This paper introduces an interactive and intelligent approach for accurate brain segmentation. A high resolution 3-Tesla magnetic resonance (MR) dataset was tested by state of the art automated algorithms as well as segmented by making use of the proposed interactive tools. The results show that the automated algorithms gave an incomplete or anatomically incorrect brain surface. About 4\% false positive and $10 \%$ false negative error rates were reported by evaluating three automated methods. The proposed approach improved the quality and accuracy of the segmented results.
\end{abstract}

\section{Introduction}

Brain extraction from neuroimages is a crucial component in neuroimage analysis systems and medical imaging applications. However, it is still a challenging task especially for high resolution data due to the image properties of small pixel size and brains with narrow sulci and dense gyri. Numerous automated or semi-automated methods have been proposed, and these methods highly depend on assumptions and parameter settings. They may give unexpected and incorrect results if some of the assumptions are not satisfied or some of the parameters are not set correctly. Afterwards, further interactive operations are followed to correct the results. The correction effort may need more manual operations and even longer time than the interactive drawing directly from the image by making use of an intelligent approach with user friendly tools.

Some applications, e.g. construction of brain atlas and generation of gold standards for segmentation algorithms, require images to be accurately segmented. An accurate delineation of sulci and gyri needs thorough anatomical knowledge. In this paper, an interactive and intelligent approach is introduced to assist the neuroanatomy expert in brain segmentation.
It is supported by several tools including efficient region of interest (ROI) based segmentation, flexible contour editor, 2D-3D correlation, and marching sulci. A $3 \mathrm{D}$ accurate brain model was built by using the introduced approach, and compared with brain segmentations of most popular automated methods.

\section{Related Work}

There are a number of methods for brain extraction from MR images proposed over a few past decades. These methods include histogram-based thresholding and morphological operations ${ }^{[1,}{ }^{2]}$, connected component analysis ${ }^{[3]}$, region growing and edge detection $^{[4]}$, voxel-based morphometry ${ }^{[5,6]}$, atlas-guided brain structure identification ${ }^{[7]}$, model-based or knowledge-guided active contour method ${ }^{[8]}$, and hybrid models ${ }^{[9,}{ }^{10]}$. In the scientific community, there are several downloadable software packages, which are widely cited by numerous papers, for brain extraction. Some of them are FMRIB software library (FSL $)^{[11]}$, BrainSuite $^{[12]}$, statistical parametric mapping $(S P M)^{[13]}$, and FreeSurfer ${ }^{[14]}$.

There are a few reviews ${ }^{[15,16]}$ attempting to evaluate these software packages by measurement of segmented whole brain or brain structures and comparison with ground truth. The selected data consisted of simulated datasets and some of real cases. BrainWeb ${ }^{[17]}$, an online interface to a 3D MR image simulated brain database (http://www.bic.mni.mcgill.ca/brainweb), is widely selected to play such a role in providing different datasets with variations of parameters (e.g. noise level, slice thickness, etc.) as well as the anatomical model (phantom). Another widely used online database is Internet Brain Segmentation Repository (http://www.cma.mgh.harvard.edu/ibsr). It provides manually guided expert segmentation results on simulated and real data for evaluation and development of segmentation methods. 


\section{Material and Method}

\subsection{Material \& Automated Method Evaluation}

A 3-Tesla (3T) high resolution MR dataset was processed by four downloaded software packages (FSL, BrainSuite, SPM, and FreeSurfer) as well as the proposed interactive approach. The pixel size of dataset is $384 \times 288 \times 176$, and its voxel size is $0.8 \times 0.8 \times 0.8 \mathrm{~mm}^{3}$.

Four software packages were executed to evaluate the automated brain extraction methods. The tested components are listed in Table 1. SPM is running with $M A T L A B$, and both FSL and FreeSurfer are running under WMware Player with default parameters.

Table 1. Automated methods

\begin{tabular}{|l|l|}
\hline 1 & FSL 4.0 (www.fmrib.ox.ac.uk/fsl) \\
\hline & $\begin{array}{l}\text { Tested components: BET (brain extraction tool) and } \\
\text { FAST (automated segmentation tool) }\end{array}$ \\
\hline & Execution time: 7 minutes \\
\hline 2 & BrainSuite 2.0 (brainsuite.usc.edu) \\
\hline & $\begin{array}{l}\text { Tested components: BSE (skull stripping), BFC (non- } \\
\text { uniformity correction), and PVC (tissue classification) }\end{array}$ \\
\hline & Execution time: 3 minutes \\
\hline 3 & SPM5 (www.fil.ion.ucl.ac.uk/spm) \\
\hline & Tested components: brain segmentation \\
\hline & Execution time: 22 minutes \\
\hline 4 & FreeSurfer 4.0.5 (surfer.nmr.mgh.harvard.edu) \\
\hline & $\begin{array}{l}\text { Tested components: 1) preprocessing and skull } \\
\text { stripping; } 2 \text { ) brain structure segmentation and surface } \\
\text { generation }\end{array}$ \\
\hline & Execution time: 15 hours \\
\hline
\end{tabular}

\subsection{Interactive Approach}

We developed four interactive supporting segmentation tools. The images generated by a skull removal software (e.g. BrainSuite) are the initial input to them. The objective is to locate the outer edges of grey matter and build a 3D surface model for the brain. The first step is to identify the sulci by ROI-based tool for segmentation and generate contours automatically for further enhancement. A flexible contour editor is used instead of a pixel editing tool for more efficient manual editing. The powerful 2D-3D correlation tool and marching sulci tool are able to assist users to easily and efficiently locate potential incorrect areas or landmarks to be edited.

3.2.1. ROI-based segmentation. The interactive segmentation tool provides the ROI-based histogram, and re-classifies the voxels with the threshold selected on the histogram graph. It basically consists of two steps: 1) draw a ROI, and then its histogram is generated and displayed; 2) select and adjust the threshold value on the histogram graph, and then the ROIs and threshold values would be recorded.

The segmented result is immediately displayed on the screen for the user to adjust the input. The user only needs to select a few ROIs to generate the segmented images and the software will merge them together and generate the contours for further enhancement.

Due to the intensity inhomogeneity between the slices, local threshold values for each ROI are required. Experience shows that the definition of ROIs and their thresholds have close values with their neighboring slices. Therefore, an efficient way to 'guess' the local ROIs and threshold values is to use the parameters selected by the user in the neighboring slices. The guess values are the default threshold values of a slice, and can be easily adjusted by the user. The new adjusted values will be learnt and applied to determination of threshold values for the subsequent slices.
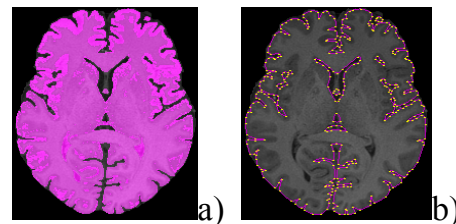

Fig. 1. Examples: a) pixel editing b) contour editing.

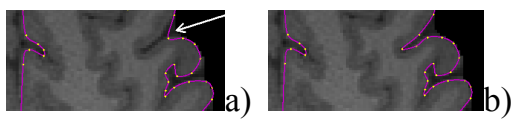

Fig. 2. An example of contour editor.

3.2.2. Flexible contour editor. The interactive contour editor provides an efficient and flexible way for the user to enhance the contours. Besides the standard operations like creation and deletion of contours, the editor provides several powerful features. It is able to 1) add and remove control points on contours (to reshape or enhance the segmentation results); 2) view original images while contouring; 3) split a contour into two (very useful to deepen the sulci and cope with the partial volume effect), or join two contours into one; 4) add labels for contours (to process simultaneously multiple objects); 5) separate brain into two hemispheres (useful to edit the interhemispheric fissure region); 6) copy a contour and paste it onto its neighboring slice(s) to speed up editing; 7) manipulate contours based on ROI (both within and outside the ROI); 8) display surface of the edited object (very helpful to generated anatomically correct structures, see also below 2D-3D correlation).

This editor is more advantageous than pixel editing. Fig. 1 gives examples of pixel editing and contour editing. For a pixel editing tool, the user is usually not able to view the original images while editing (shown 
in Fig. 1(a)), and for contour editing, the original image is almost not covered by contours and the user still has a good view of the image. Fig. 2 gives another example. The sulcus pointed by the arrow (Fig. 2(a)) is under-segmented and it can be easily corrected by adding a few more points (Fig. 2(b)).

3.2.3. 2D-3D correlation. The $2 \mathrm{D}-3 \mathrm{D}$ correlation tool greatly facilitates generation of correct anatomy. In general, on 2D slices it is very difficult to determine the correct anatomy, mainly due to the partial volume effect and 3D course of structures. The 3D display enables identification of problems, such as unrealistic shapes, too shallow sulci, bridges between gyri, bumps on cortical surface, and missing or incomplete sulci, among others. Then, the region to be corrected is identified on the cortical surface and it is mapped on the original 2D image for editing by the contour editor. Conversely, only location on a 2D slice can be mapped on the 3D surface for inspection.

The brain surface is rendered as hundreds of thousands of triangles lighted and shaded by Open $G L^{[18]}$, which is widely used in $3 \mathrm{D}$ rendering applications. The triangles are generated by the Marching Cubes ${ }^{[19]}$ which decides whether each isotropic voxel is inside or outside the surface.

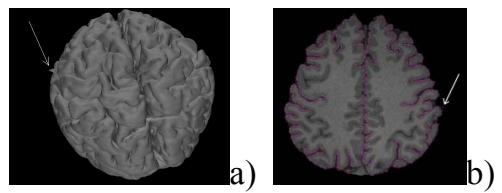

Fig. 3. Spatial correlation of 3D with 2D.

a) position on $3 \mathrm{D}$ display; b) correlated $2 \mathrm{D}$ position.

Usually the 3D surface rendering display is more obvious than 2D image display to show the shapes of anatomic structures, as shown in Fig. 3. While mouse is clicked on a $3 \mathrm{D}$ display, only one point $p(x, y)$ is provided (pointed by the arrow in Fig. 3(a)). The correlated 2D position of the point needs a few steps to be located. Firstly, the triangles which are within the mouse click are identified, and the one nearest to the user is selected. If the selected triangle is not small enough $(<0.1 \mathrm{~mm})$, then it will be equally divided into 3 smaller triangles and the one within mouse click is selected. The selected triangle will be repeatedly divided into smaller triangles until the selected triangle is small enough. The center position of final selected triangle is considered as the coordinate of the selected point on the $3 \mathrm{D}$ display, as well as the coordinate on $2 \mathrm{D}$ image. The user can focus on this position to correct the false segmentation as shown in Fig. 3(b).

3.2.4. Marching sulci. The sulcus is one of the most difficult areas to be properly determined. The slight inaccuracy is often missed, or sometimes misled by pixels with similar intensities. In order to highlight such kind of slight inaccuracy, an interactive tool is implemented to calculate the curvatures for each vertex of triangles and paint the different colors for the vertices. The mean curvatures are calculated by making use of Visualization Toolkit $(V T K)^{[20]}$ library and non-linear diffusion is applied ${ }^{[21]}$. The calculated results are mapped to predefined colors. Fig. 4 shows examples of surface curvatures.

The convex surfaces have higher curvature values (positive), while the concave surfaces have lower curvature values (negative). The flat areas have curvature values close to zero. A threshold value $\theta$ is selected by the user to determine curvature highlighting. Fig. 4(a) shows an example of surface curvatures with $\theta=0.2$. That means, the surfaces with curvature values of $+0.2(-0.2)$ or higher (lower) are painted with blue (red) color and the surfaces with curvature values between -0.2 and +0.2 are painted with grey color. Fig. 4(b) gives an example with $\theta=2$ and Fig. 4(c) shows the zoom-in image of Fig. 4(b). The surface areas highlighted in such way have likely wrong segmentation.
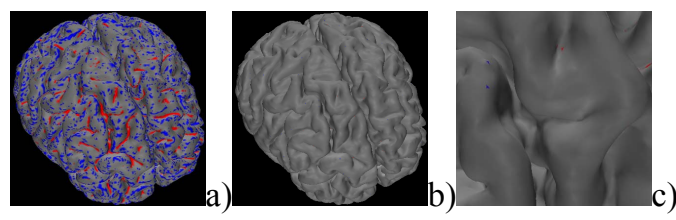

Fig. 4. Examples of surface curvatures. a) $\theta=0.2$; b) $\theta=2$; and c) zoom-in of b).

\section{Results}

The automated methods were tested on a PC with 2.4 GHz CPU running Windows XP. The interactive tools were implemented in $\mathrm{C}++$ programming language, linked with VTK library and the Marching Cubes algorithm for 3D surface rendering and curvature calculation, running and testing on the same computer.

Fig. 5 lists the results generated by FSL, BrainSuite, and $S P M$ on 2D slice, 3D far view, and 3D close view of surface rendering. Fig. 6 shows the results from pial surface generated by FreeSurfer in coronal orientation. FSL divides the brain into grey matter (GM), white matter (WM), and cerebrospinal fluid (CSF). The shown results in Fig. 5(a) are the combination of GM and WM. BrainSuite generates not only the results of GM, WM, and CSF, but also their partial pixels. The results shown in Fig. 5(b) include the partial pixels of GM/WM but exclude the partial pixels of GM/CSF. SPM gives the probabilities of GM, WM, and CSF. The results (Fig. 5(c)) had 90\% confidence of segmented GM and WM. FreeSurfer transformed the 
original images into $256 \times 256 \times 256$. In such case, it requires to transform the segmented results back to the original images.

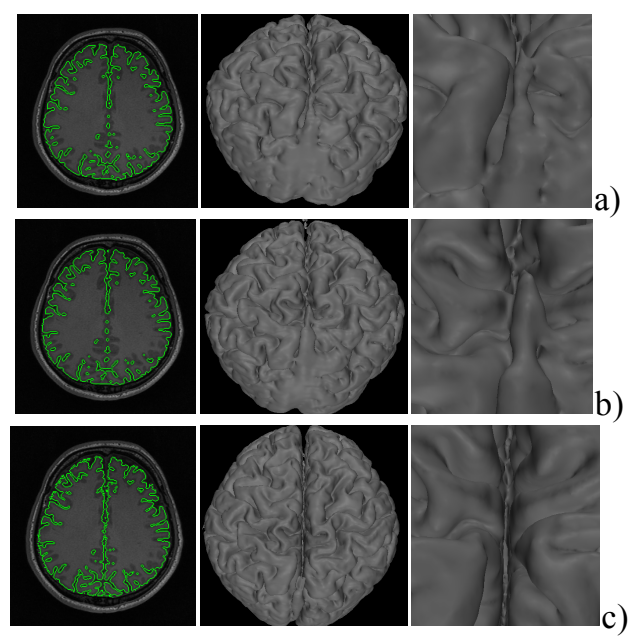

Fig. 5. 2D segmented result (left), far 3D view (mid), and close 3D view (right): a) FSL; b) BrainSuite; c) SPM.

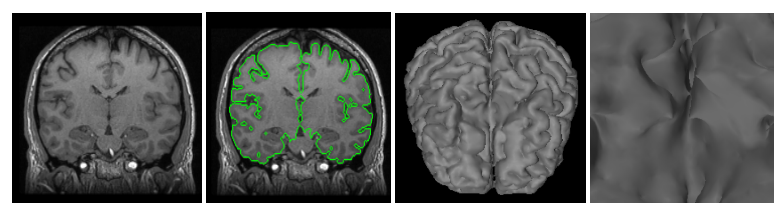

Fig. 6. Results of FreeSurfer, from left to right: original coronal slice, results for $2 D$ slice, far view $3 D$ surface rendering, and close view 3D surface rendering.

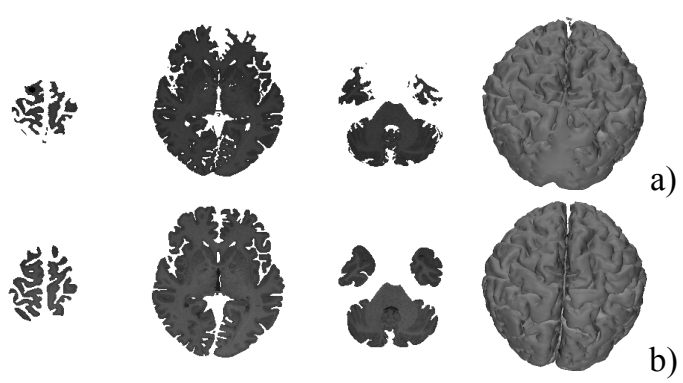

Fig. 7. Results of a) automated brain extraction, and b) applying interactive tools.

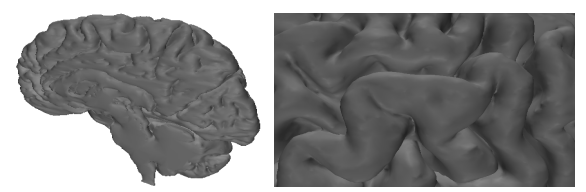

Fig. 8. Segmented 3D: a) far view, and b) close view.

The results from an automated method are shown in Fig. 7(a). The further gyri segmentation was done by a neuroanatomy expert with the interactive tools and the results are shown in Fig. 7(b). The first three 2D images are superior slice, mid slice, and inferior slice. The 3D images are the outcome of surface rendering based on the segmented 2D images. Fig. 8 gives the results of one hemisphere and a close view of sulci.
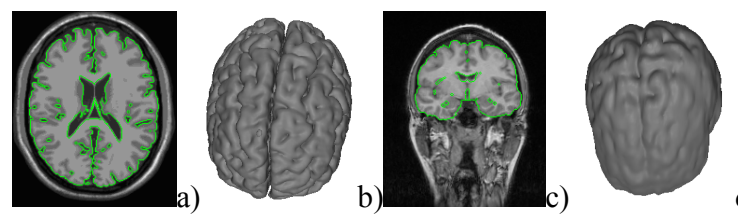

Fig. 9. 2D slice with ground truth and 3D view. $\operatorname{Brain} W e b(\mathbf{a}, \mathrm{b})$ and $I B S R(\mathbf{c}, \mathrm{d})$.

Table 2 compares the results from the automated brain extraction programs and the results by applying interactive tools. Volume is the size of segmented brain. True positive (TP) with success rate, and false positive (FP) and false negative $(F N)$ with their error rates are calculated for each automated methods. FreeSurfer is not included into the table because it transformed the images and its results are difficult to be compared with original images.

Table 2. Comparison of results from the automated methods and the interactive approach*

\begin{tabular}{|l|c|c|c|}
\hline & FSL & BrainSuite & SPM \\
\hline Volume $\left(\mathrm{cm}^{3}\right)$ & 1140.85 & 1119.55 & 1106.71 \\
\hline$($ Dice Idx $\%$ & 93.37 & 92.59 & 93.15 \\
\hline $\mathrm{TP} \mathrm{cm}^{3}$ & 1089.61 & 1070.7 & 1071.24 \\
\hline$($ TP rate $) \%$ & 91.32 & 89.73 & 89.78 \\
\hline FP cm & 51.25 & 48.85 & 35.46 \\
\hline$($ FP rate $\%$ & 4.29 & 4.09 & 2.97 \\
\hline FN cm & 103.61 & 122.51 & 121.97 \\
\hline (FN rate $\%$ & 8.68 & 10.27 & 10.22 \\
\hline
\end{tabular}

${ }^{*}$ Volume of interactive approach is $1193.22 \mathrm{~cm}$

\section{Discussion and Conclusions}

Currently the most automated methods reported their accuracy by comparison of segmented results with some ground truth. This is done by pixel-by-pixel comparison without applying any anatomical knowledge. Therefore, if the ground truth is only visually correct in images but not anatomically correct in knowledge, the comparison may only make sense in pure image processing terms. Fig. 9 shows examples of original 2D slices with segmented results and 3D surface rendering, from a BrainWeb phantom (modality: T1, noise level: $0 \%$, slice thickness: $1 \mathrm{~mm}$, intensity non-uniformity: $0 \%$ ), and $I B S R$ (case 1_24), respectively. Each slice of the images shows the ground truth segmentation (Fig. 9(a) and Fig. 9(c)). However, unfortunately it gives an incomplete or anatomically incorrect view by rendering the surface in 3D (Fig. 9(b) and Fig. 9(d)).

The proposed approach provides a way for accurate brain segmentation by making use of user friendly tools with rich features and several advantages: 1) ROI-based segmentation. The selection of current segmented slice is propagated to neighboring slices. It 
greatly speeds up process of segmentation. 2) Flexible contour editor. A contour can be split into two different contours, and the contours can be merged into one. It is helpful for a gyrus which is a single contour in one slice but has more contours in another slice. 3) 2D-3D correlation, a very user friendly tool to locate exact positions on different views. 4) Marching sulci, an efficient way to highlight the areas potentially incorrect, and is very useful to generate highly accurate results.

This approach has some limitations such as being time consuming for the dataset with large number of slices. It is still not able to edit the surface directly on the $3 \mathrm{D}$ view. Combination of our approach with voxel editing would speed up some operations.

In conclusion, an interactive approach with several interactive supporting tools is introduced in this paper. These interactive segmentation tools play an indispensable role in accurate brain extraction from volumetric MR neuroimages due to partial volume effect, artifacts, noise, and intensity inhomogeneity. The results showed a significant improvement for accurate brain segmentation.

\section{References}

[1] Shan, Z.Y., Yue, G.H., and Liu, J.Z., Automated Histogram-Based Brain Segmentation in T1-Weighted Three-Dimensional Magnetic Resonance Head Images. Neuroimage, 2002. 17(3): pp. 1587-1598.

[2] Stokking, R., Vincken, K.L., and Viergever, M.A., Automatic Morphology-Based Brain Segmentation (MBRASE) from MRI-T1 Data. Neuroimage, 2000. 12(6): pp. 726-738.

[3] Lemieux, L., Hagemann, G., Krakow, K., and Woermann, F.G., Fast, accurate, and reproducible automatic segmentation of the brain in T 1-weighted volume MRI data. Magnetic Resonance in Medicine, 1999. 42(1): pp. 127-135.

[4] Xuan, J., Adali, T., and Wang, Y., Segmentation of magnetic resonance brain image: integrating regiongrowing and edge detection. Image Processing, 1995. Proceedings., International Conference on, 1995. 3.

[5] Ashburner, J. and Friston, K.J., Voxel-Based Morphometry - The Methods. Neuroimage, 2000. 11(6): pp. 805-821.

[6] Yu, A., Li, K., Li, L., Shan, B., Wang, Y., and Xue, S., Whole-brain voxel-based morphometry of white matter in medial temporal lobe epilepsy. European Journal of Radiology, 2008. 65(1): pp. 86-90.

[7] Akselrod-Ballin, A., Galun, M., Gomori, M.J., Basri, R., and Brandt, A., Atlas guided identification of brain structures by combining 3D segmentation and SVM classification. LNCS, 2006. 4191: pp. 209.
[8] Shan, Z.Y., Ji, Q., Gajjar, A., and Reddick, W.E., A knowledge-guided active contour method of segmentation of cerebella on MR images of pediatric patients with medulloblastoma. Journal of Magnetic Resonance Imaging, 2005. 21(1): pp. 1-11.

[9] Rehm, K., Schaper, K., Anderson, J., Woods, R., Stoltzner, S., and Rottenberg, D., Putting our heads together: a consensus approach to brain/non-brain segmentation in T1weighted MR volumes. Neuroimage, 2004. 22(3): pp. 12621270 .

[10] Tu, Z. and Toga, A.W., Towards Whole Brain Segmentation by a Hybrid Model. LNCS, 2007. 4792: pp. 169.

[11] Smith, S.M., Jenkinson, M., Woolrich, M.W., Beckmann, C.F., Behrens, T.E.J., Johansen-Berg, H., Bannister, P.R., De Luca, M., Drobnjak, I., and Flitney, D.E., Advances in functional and structural MR image analysis and implementation as FSL. Neuroimage, 2004. 23: pp. 208-219.

[12] Shattuck, D.W. and Leahy, R.M., BrainSuite: An automated cortical surface identification tool. Medical Image Analysis, 2002. 6(2): pp. 129-142.

[13] Friston, K.J., Introduction: Experimental design and statistical parametric mapping. Hum brain function, 2003. 2.

[14] Dale, A.M., Fischl, B., and Sereno, M.I., Cortical Surface-Based Analysis I. Segmentation and Surface Reconstruction. Neuroimage, 1999. 9(2): pp. 179-194.

[15] Boesen, K., Rehm, K., Schaper, K., Stoltzner, S., Woods, R., Luders, E., and Rottenberg, D., Quantitative comparison of four brain extraction algorithms. Neuroimage, 2004. 22(3): pp. 1255-1261.

[16] Klauschen, F., Goldman, A., Barra, V., MeyerLindenberg, A., and Lundervold, A., Evaluation of automated brain MR image segmentation and volumetry methods. Hum Brain Mapp, 2008. In Press.

[17] Cocosco, C.A., Kollokian, V., Kwan, R.K.S., and Evans, A.C., BrainWeb: Online interface to a 3D MRI simulated brain database," Neuroimage, vol. 5, no. 4, 1997.

[18] OpenGl, A.R.B., Shreiner, D., Woo, M., Neider, J., and Davis, T., OpenGL Programming Guide: The Official Guide to Learning OpenGL, Version 2. 2005, Boston: AddisonWesley Professional.

[19] Lorensen, W.E. and Cline, H.E., Marching cubes: A high resolution 3D surface construction algorithm. Proceedings of the 14th annual conference on Computer graphics and interactive techniques, 1987: pp. 163-169.

[20] Schroeder, W., Martin, K., and Lorensen, B., The Visualization Toolkit An Object-Oriented Approach To 3D Graphics, Kitware. Inc. publishers, 2004. 2.

[21] Jin, J.S., Wang, Y., and Hiller, J., An adaptive nonlinear diffusion algorithm for filtering medical images. Information Technology in Biomedicine, IEEE Transactions on, 2000. 4(4): pp. 298-305. 